\title{
Umweltrisiken verstehen
}

\author{
In Entwicklungsländern tätige Banken sind mit ähnlichen Umweltrisiken \\ konfrontiert wie in Industrieländern, namentlich Kredit- und Marktrisiken \\ sowie Reputationsrisiken. Verschiedene Finanzinstitutionen verfolgen jedoch \\ spezielle Strategien im Umgang mit diesen Risiken. Für Geschäftsbanken ste- \\ hen primär kommerzielle Ziele im Vordergrund, für Entwicklungsbanken die \\ Förderung einer nachhaltigen Entwicklung. Unabhängig von den unterschied- \\ lichen Zielen, ist die ideale Grundlage für die Kontrolle von Umweltrisiken \\ die selbe: Ein Verständnis der Effekte, einschließlich externer Effekte, welche \\ von den finanzierten Projekten oder Unternehmen ausgehen.
}

B ankdienstleistungen belasten die Umwelt nicht direkt. Der Finanzsektor hat in erster Linie indirekte Umweltwirkungen - über Unternehmen und Projekte, denen er Kapital zur Verfügung stellt. Sind diese Umweltwirkungen bedeutend oder haben sie finanzielle Folgen für ein Unternehmen, können aus Umweltrisiken eines Schuldners Geschäftsrisiken der finanzierenden Bank werden. Wissen um indirekte Effekte finanzierter Projekte und Unternehmen ist daher aus unterschiedlichen Anreizen für unterschiedliche Akteure auf Finanzmärkten in Entwicklungsländern wichtig. Für Geschäftsbanken steht die Kontrolle von Umweltrisiken im Vordergrund. Entwicklungsbanken erlaubt es zusätzlich eine Beurteilung, ob finanzierte Projekte zu einer nachhaltige Entwicklung beitragen.

\section{Umweltrisiken für Banken}

1. Umweltrisiken als Kreditrisiken. Ölkatastrophen, illegale Holzexporte oder etwa die Ver- schmutzung von Gewässern können Bußen oder unerwartete Investitionen nach sich ziehen, welche die Kreditwürdigkeit des Unternehmens verschlechtern. Beispiele: Die brasilianischen Umweltbehörden verfügten Ende der 1990er-Jahre Bußen von jährlich zehn bis zwölf Millionen US Dollar (1). Petrobras, ein brasilianisches Ölunternehmen, wurde nach Unfällen in Raffinerien mit Bußgeldern in Höhe von insgesamt und 146 Millionen US Dollar belegt. Diese Bußgelder gehören zu den bisher höchsten in Lateinamerika (2). Eine Verschlechterung der Kreditwürdigkeit ist auch die Folge, wenn Schadstoffbelastungen den Wert eines belehnten Grundstücks verringern. Umweltrisiken als Kreditrisiken dürften in Entwicklungsländern mit der weltweiten Harmonisierung von Umweltstandards in Zukunft an Bedeutung zunehmen (3).

2. Umweltrisiken als Marktrisiken. Kapitalmärkte reagieren auf Informationen über Umweltwirkungen von Unternehmen in Entwicklungsländern. Dies gilt nicht nur für multinationale, sondern auch für lokal tätige und an lokalen Börsen notierte Un-

\begin{tabular}{|l|l|l|c|c|}
\hline \multicolumn{4}{|c|}{ Tabelle l: Umweltstrategien von Banken in Entwicklungs- und Schwellenlindern } \\
\hline \multicolumn{1}{|c|}{ Region } & Lateinamerika & Afrika & $\begin{array}{c}\text { Zentral- und } \\
\text { Osteuropa }\end{array}$ & Asien \\
\hline $\begin{array}{l}\text { Internationale Ent- } \\
\text { wicklungsbanken }\end{array}$ & & & \\
\hline $\begin{array}{l}\text { Internationale } \\
\text { Geschäftsbanken }\end{array}$ & & & & \\
\hline $\begin{array}{l}\text { Regionale und } \\
\text { nationale Banken }\end{array}$ & & & & \\
\hline $\begin{array}{l}\text { MFI (Mikrofinanz- } \\
\text { Institution) }\end{array}$ & & & & \\
\hline
\end{tabular}

Quelle: Ecofact, 2002 ternehmen. Kapitalmärkte reagieren negativ auf Presseberichte über Bürgerproteste und scheinen umgekehrt die Ankündigung neuer, umweltfreundlicherer Technologien zu belohnen (4).

3. Umweltrisiken als Reputationsrisiken. Für die Mehrzahl der in Entwicklungsländern aktiven Finanzdienstleister geben Reputationsrisiken den Ausschlag dafür, sich mit Umweltrisiken zu beschäftigen. Hermann Mulder von ABN AMRO beispielsweise glaubt, dass für Banken Umweltschäden primär Reputationsrisiken bergen und Kreditrisiken von untergeordneter Bedeutung sind (5).

\section{Umweltrisikomanagement}

Anders als in Industrieländern kennen Banken in Entwicklungsländern Umweltmanagementsysteme noch kaum. Bis heute haben rund 50 Finanzinstitute aus Entwicklungsländern die UNEP-Bankenerklärung ratifiziert, jedoch nur selten weitere Schritte unternommen (6).

Wie Abbildung 1 zeigt, sind Entwicklungsbanken im Umgang mit Umweltaspekten am weitesten fortgeschritten. Die Schattierung der Felder gibt den Stand der Entwicklung an. Schwarz: weit entwickelt; Hellgrau: wenig entwickelt. Zwischen einzelnen Ländern, Regionen und Institutionen bestehen bedeutende Unterschiede. Die Weltbank analysiert seit Ende der 1980er Jahre die Umweltwirkungen ihrer Projekte. Heute sind derartige Analysen bei sämtlichen regionalen Entwicklungsbanken üblich, wobei sich viele an Weltbankstandards orientieren (7).

Lokale kommerzielle Banken in Entwicklungsländern berïcksichtigen Umweltaspekte in ihrer Tätigkeit noch kaum. Zwar besteht ein gewisser Druck seitens multilateraler Organisationen. Der Entwicklung wirksamer Umweltmanagementsystem stehen aber oft knappe Ressourcen und fehlende Erfahrung im Weg. Ähnliches gilt für Mikrofinanzorganisationen, deren Bedeutung in ärmeren Ländern zunimmt. Die Vergabe von Mikrokrediten ist zwar ein Bankgeschäft wie jedes andere. Jedoch liegen beispielsweise in der großen Anzahl kleinster Kredite oder in der nicht alphabetisierten Kundschaft neue Risiken, deren Kontrolle neue Ansätze erfordert.

\section{Fazit: Zwei Thesen}

Erstens: Eine Bank, welche Umweltkriterien in ihre Finanzierungsentscheide integriert, kontrolliert ihre Risiken besser. Umweltrisikomanagement zahlt sich aus. Zweitens: Die Berücksichti- 


\section{Drei Fallbeispiele}

Fallbeispiel 1: ABN AMRO, eine der zehn größten europäischen Banken, formulierte 1992 erstmals eine Umweltpolitik. Ende der 1990er Jahre löste der Rummel um Brent Spar eine erneute interne Debatte aus, welche zu einem Mentalitätswandel führte. Die Wahrnehmung von Reputationsrisiken wurde differenzierter.

ABN AMRO geriet verschiedentlich in die Kritik von NGOs, welche auf die Umweltwirkungen von Finanzierungen in den Sektoren Bergbau und Forstwirtschaft in Entwicklungsländern hinwiesen. Als Reaktion verabschiedete ABN AMRO 2001 eine Geschäftspolitik, welche die „Nachhaltigkeit“ von Forstwirtschaftsprojekten sicherstellen soll. 2002 wurden Richtlinien für die Finanzierung von Bergbauprojekten erarbeitet, ähnliche Grundsätze für die Ölindustrie werden diskutiert. Im internationalen Kreditgeschäft geschieht eine Umweltrisikoprüfung, welche sich an der lokalen Gesetzgebung sowie den Grundsätzen von Weltbank und OECD orientiert. Ökologische und soziale Aspekte der Projektfinanzierung werden anhand der Equator Principles geprüft.

Quellen: www.abnamro.com, www.equator-principles.com

Fallbeispiel 2: DBP, die Entwicklungsbank der Philippinen, ist ein wichtiger Kreditgeber der philippinischen Industrie. Das Thema Umwelt gewann an Bedeutung, als die Weltbank 1992 eine Kreditlinie mit der Auflage verband, eine Umweltpolitik für das Kreditgeschäft zu entwickeln.

Nach und nach löste das Engagement des Bankmanagements den Druck der Weltbank als treibende Kraft hinter der Entwicklung eines Umweltmanagementsystems (UMS) ab. Das UMS ist heute in alle Geschäftsbereiche der Bank integriert und wurde vor kurzem nach ISO 14001 zertifiziert. Als schwierig erwies sich anfänglich die Beurteilung von Kunden nach Umweltkriterien. Heute nimmt die DBP über die Kreditprüfung hinaus eine Beraterrolle wahr, in dem sie ihre Kunden im Umgang mit Umweltaspekten unterstiitzt.

Quelle: www.devbankphil.com.ph

Fallbeispiel 3: Eine der größten Mikrofinanz-Organisationen, die Grameen Bank in Bangladesh, gewährt den Armen Finanzdienstleistungen. Teil der Entwicklungsziele der Bank ist, dass sich Kreditnehmer eine Reihe Prinzipien zu Disziplin, Gesundheitsfürsorge, Unabhängigkeit, Ausbildung, Solidarität und Umweltschutz aneignen. Effekt: Bis heute haben sich mehr als 2.7 Millionen Kunden mit der Regel befasst „Wir sollen immer unsere Kinder und die Umwelt sauber halten". Quelle: www.grameen-info.org

Countries, Geneva 2002.

(4) World Bank Development Research Group: Capital market responses to environmental performance in developing countries, 1998.

(5) ABN AMRO (Ed.): Environment report 1998-2000, 2001.

(6) www.unepfi.net

(7) World Bank (Ed.): Environment matters, 2001.

Diese beiden Thesen umschreiben den Beitrag, den Finanzdienstleister zu einer nachhaltige Entwicklung leisten können.

\section{Anmerkungen}

(1) Nolet, G.: Environmental Enforcement in Latin America and the Caribbean. In: International Network for Environmental Compliance and Enforcement (INECE), Fifth International Conference on Environmental Compliance and Enforcement, 1998.

(2) Gracer, J. B.: Green Risks on the Rise. In: Latin Finance, Sept. 2000.

(3) Utting, P.: Towards Corporate Environmental Responsibility. In: United Nations Research Institute for Social Development (Ed.): The Greening of Business in Developing
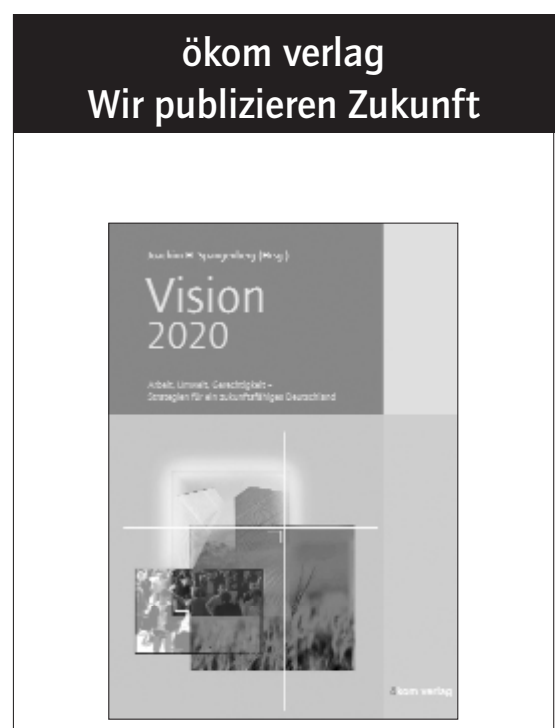

Vision 2020 - Arbeit, Umwelt, Gerechtigkeit

\section{Strategien für ein zukunftsfähiges Deutschland}

Für eine ökologisch und sozial nachhaltige Gesellschaft müssen wir neue Strategien entwickeln - irgendwo zwischen der Vision vom "guten Leben" und den ökonomischen Zwängen der Realität. Die Hans-Böckler-Stiftung ließ in dem interdisziplinären Projekt "Arbeit und Ökologie" verschiedene Nachhaltigkeitskonzepte unter die Lupe nehmen. Das Buch fasst die Ergebnisse zusammen und entwickelt daraus Empfehlungen für Deutschlands Weg in die Zukunft.

Joachim Spangenberg (Hrsg.), ökom Verlag,

München 2003, 300 Seiten,

23,- € (zzgl. Versandkosten) ISBN 3-936581-07-X

\section{Die Autoren}

François Mercier betreut bei Ecofact das Projekt "Environment and Finance in Developing Countries". Oliver Zenklusen ist Berater bei Ecofact und promoviert über die ökonomischen Effekte von Naturkatastrophen in Entwicklungsländern.

Kontakt: ECOFACT AG, Stampfenbachstrasse 42, CH-8006 Zürich, Tel. 0041 (0)1-3506060,

E-Mail: zenklusen@ecofact.ch

\section{Erhältlich bei} CONSODATA ONE-TO-ONE Semmelweisstraße 8, D-82152 Planegg Fon $++49 /(0) 89 / 857$ 09-155 Fax $++49 /(0) 89 / 857$ 09-131 kontakt@oekom.de 
(c) 20I0 Authors; licensee IÖW and oekom verlag. This is an article distributed under the terms of the Creative Commons Attribution Non-Commercial No Derivates License (http://creativecommons.org/licenses/by-nc-nd/3.o/), which permits unrestricted use, distribution, and reproduction in any medium, provided the original work is properly cited. 